Pacific Journal of Mathematic

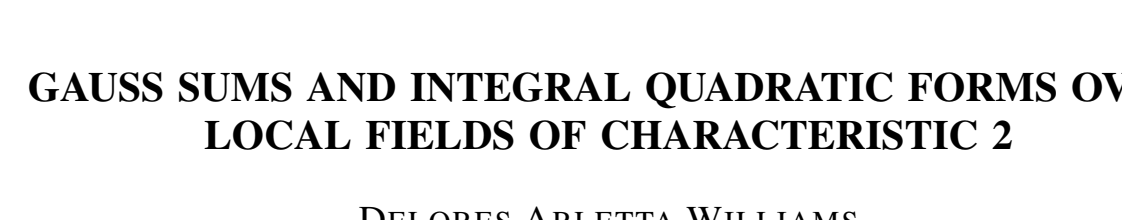




\title{
GAUSS SUMS AND INTEGRAL QUADRATIC FORMS OVER LOCAL FIELDS OF CHARACTERISTIC 2
}

\author{
Delores A. Williams
}

\begin{abstract}
The theory of Gauss sums is developed for integral quadratic forms over a local field of characteristic 2, and Gauss sums are used to characterize these forms. For a character $\chi$ and an integral lattice $L$, the Gauss sum $\chi(L)$ is either zero, a nonnegative power of two, or the negative of a positive power of two. Gauss sums alone characterize the integral equivalence classes for modular lattices. For arbitrary lattices, other invariants are required.
\end{abstract}

The classification given in this paper is an alternate to the one by C.-H. Sah [6]. The notation and terminology of [6] is used except when stated to the contrary. O. T. O'Meara [4] used Gauss sums to characterize local integral quadratic forms over a field of characteristic not 2 , and $R$. Jacobowitz [3] classified hermitian forms over the integers of a local field of characteristic not 2 by Gauss sums. When needed, results from these papers are referred to when the proofs hold for the characteristic 2 case.

After a few preliminaries, we introduce Gauss sums and prove some results for Gauss sums of lines and planes that will in turn be used to study more complicated lattices. In Theorem 5.4 we show that Gauss sums alone are sufficient to characterize modular lattices, and in Theorem 4.2 we show that for nondefective lattices only a finite number of Gauss sums need be considered.

1. Preliminaries. Throughout this paper $k$ denotes a local field of characteristic 2 with fixed prime element $\pi$, ring of integers 0 , and residue class field of order $2^{f}$. We let $\Omega$ denote a complete set of representatives for the residue class field. We refer the reader to [6] for a discussion of the Arf invariant $\Delta V$ for a quadratic space $V$ and the additive group $\mathscr{P} \Omega$. As in [6] we let $\{0, \lambda\}$ be a fixed set of representatives of $\Omega / \mathscr{P} \Omega$. The letter $e$ always denotes a unit of $k$. For a nonnegative integer $s, H_{s}$ and $H_{s}^{\prime}$ denote $s$ hyperbolic lattices.

Let $L$ be a lattice. $K(L)=\{x \in L \mid\langle x, y\rangle=0$ for all $y \in L\}$. If $K(L)=0$, then $L$ is nondefective. Otherwise, it is defective. We assume that if $x \in K(L)$ and $Q(x)=0$, then $x=0$.

We now state some lemmas and definitions from [6] in the form in which they are used in this paper. 
LEMMA 1.1. Let $L$ be a lattice with $(q L) \mathfrak{D}=\pi^{u} \mathfrak{D}$.

(1) If $\operatorname{rank}{ }_{{ }_{2}} q(L)=1$, then $L=K(L)$ and $q(L)=e \pi^{u} \mathrm{D}^{2}$ with $e$ a unit.

(2) If $\operatorname{rank}_{{ }_{0} 2} q(L) \geqq 2$, then

$v=\min \{\operatorname{ord} a \mid a \in q(L)$ and ord $a \equiv u+1 \bmod 2\}$ is a rational integer with $u<v$. If, in addition, $L \neq K(L), v \leqq s+1$ with $s L=\pi^{s} \mathrm{v}$. Moreover, for any unit $e$ with $e \pi^{u} \in Q(L), q(L)=e\left(\pi^{u} \mathfrak{D}^{2}+\pi^{v} \mathfrak{D}^{2}\right)$; and there is at least one such $e$.

If more that one lattice is under consideration, the invariants $u$ and $v$ for $L$ are denoted by $u_{L}$ and $v_{L}$ respectively.

As in [6], for a rational integer $i, u(i)$ and $v(i)$ denote the $u$ and $v$ of Lemma 1.1 for the lattice $L(i) \otimes H_{i}$.

LEMMA 1.2. Let $L$ be an $i$-modular lattice such that $\operatorname{rank} L \geqq 4$ and $q(L)=e\left(\pi^{u} \mathfrak{D}^{2}+\pi^{v} \mathfrak{D}^{2}\right)$ with $u<v \leqq i+1, u+v \equiv 1 \bmod 2$, and $e \pi^{u} \in Q(L)$ (as in Lemma 1.1). Then $L \simeq\left(\begin{array}{c}\pi^{i} \\ e \pi^{v}\end{array} e^{-1} \pi^{2 i-u} \delta+e^{-1} \pi^{v} a^{2}\right) \oplus$ $\left(\begin{array}{c}\pi^{i} \\ e \pi^{v} \quad e^{-1} \pi^{2 i-v} \delta^{\prime}\end{array}\right) \oplus H$ with $\delta, \delta^{\prime} \in\{0, \lambda\}, H$ i-hyperbolic or 0 , and $\delta^{\prime}=0$ when $v=i+1$. Moreover, $q(L)=Q(L)$.

For a canonical decomposition $L=\left(\sum_{i=0}^{t} L_{i}\right) \oplus K(L), s(i)$ always denotes $s\left(L_{i}\right)$.

Definition 1.3. Let $L=\left(\sum_{i=0}^{t} L_{i}\right) \oplus K(L)$ be a canonical decomposition. Define $I=s(0)$ and $T=s(t)+\max \left\{u^{\prime}, v^{\prime}\right\}$, where

$$
u^{\prime}= \begin{cases}u_{K(L)}-u(s(t)) & \text { if } L \text { is defective } \\ 0 & \text { otherwise }\end{cases}
$$

and

$$
v^{\prime}=\left\{\begin{array}{ll}
v_{K(L)}-v(s(t)) & \text { if } \operatorname{rank}_{{ }^{2} 2} q(K(L))=2 \\
0 & \text { otherwise }
\end{array},\right.
$$

and defined $R=(r(I), r(I+1), \cdots, r(T))$ where

$$
r(j)=\left\{\begin{array}{ll}
\operatorname{rank} L_{i} \text { if } s(i)-j \text { for some } i \in\{0, \cdots, t\} \\
0 \quad \text { otherwise }
\end{array} .\right.
$$

Then $L$ is said to be of type $(I, T, R)$ and length $(t+1)$.

Definition 1.4. For $L \neq K(L)$, let $L=\left(\sum_{i=0}^{t} L_{i}\right) \oplus K(L)$ be a canonical decomposition of $L$. If $s(0), \cdots, s(t)$ are consecutive integers, rank $L_{i} \geqq 8$, and $u^{\prime}=v^{\prime}=0$, then $L_{i}$ is a normal lattice of type $(s(0), s(t), R)$ and $R=\left(\operatorname{rank} L_{0}, \cdots\right.$, rank $\left.L_{t}\right)$. Observe that 
the concept of normal is independent of the canonical decomposition.

LemMa 1.5. Let $L=\sum_{i=0}^{t} L_{i}$ and $M=\sum_{i=0}^{t^{\prime}} M_{i}$ be canonical decomposition of the non-defective lattices $L$ and $M$ respectively. If $L$ and $M$ are of the same type, then $t=t^{\prime}, \operatorname{rank} L_{i}=\operatorname{rank} M_{i}$, and $s_{L}(i)=s_{M}(i)$.

2. Characters, A character of $\mathfrak{o}$ is a map $\chi$ from $\mathfrak{o}$ into the complex numbers such that $\chi(a+b)=\chi(a) \chi(b)$ for $a, b \in \mathfrak{p}$, and $\chi(\mathrm{o}(r))=1$ for some nonnegative rational integer $r$. Let $m(\chi)$ denote the smallest $r \geqq 0$ such that $\chi(\mathfrak{o}(r))=1$. Then $\mathfrak{o}(m(\chi))$ is called the maximal support of $\chi$ and $m(\chi)$ is called the maximal support ordinal of $\chi$. When a single character $\chi$ is being considered, we use $m$ for the maximal support ordinal of $\chi$ without stating that $m=m(\chi)$.

Note that if $\chi$ is a character and $a \in \mathfrak{v}$, then $\chi(\alpha)= \pm 1$. In fact, the image of $\chi$ is $\{-1,1\}$ except when $m(\chi)=0$.

The set of all characters of $\mathfrak{v}$, denoted by $X$, together with the operation of function multiplication is a group. For $r \geqq 0$ define $X(r)=\{\chi \in X \mid \chi(\mathrm{o}(r))=1\}=\{\chi \in X \mid m(\chi) \leqq r\} . \quad X(r)$ is a subgroup of $X$ and will play an important role in the study of Gauss sums.

For each $r \geqq 0$ there is a one-to-one correspondence between $X(r)$ and the set of group characters on $\mathrm{v} / \mathrm{o}(r)$. This permits us to obtain information about $X(r)$ from the theory of group characters. (For details see [3].)

3. Gauss sums, A lattice $L$ is said to be integral if $Q(L) \subseteq 0$. If $L$ is integral, then $q(L) \subseteq \mathfrak{D}$, since for $x, y \in L,\langle x, y\rangle=Q(x)+$ $Q(y)+Q(x+y) \in \mathcal{D}$. All lattices will be assumed to be integral. This causes no loss of generality in studying the classification problem.

For $r \geqq 0, L(r)$ will denote $\pi^{r} L$ and a summation over $x \bmod$ $L(r)$ will mean that $x$ runs over a complete set of representatives for the additive group $L / L(r)$. The order of $L / L(r)$ is $2^{f r(\operatorname{rank} L)}$.

For a lattice $L$ and a character $\chi \in X(r), \chi\left(L ; \pi^{r}\right)=\sum_{x \bmod L(r)} \chi(Q(x))$ is called a Gauss sum. Write $\chi(L)$ for $\chi\left(L ; \pi^{m}\right)$. Since $L$ is integral and $L / L(r)$ is finite, all elements of $Q(L)$ are in the domain of $\chi$ and each Gauss sum is a finite sum of ones and negative-ones.

For a plane $P \cong\left(\begin{array}{c}b \\ a c\end{array}\right)$ (or a line $\left.P \cong(a)\right)$, we will sometimes write $\chi\left(\begin{array}{c}b \\ a c\end{array}\right)$ (or $\chi(a)$ ) for $\chi(P)$.

In view of [4, Propositions 1 and 2], it is clear that a great 
deal can be learned about Gauss sums by studying $\chi(L)$ when $L$ is a line or plane. Our investigation can be further simplified by noting:

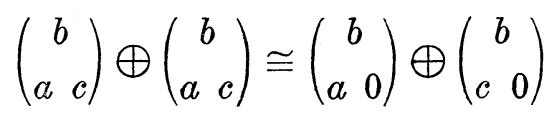

and

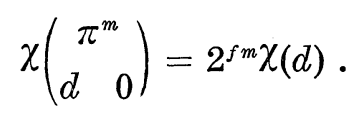

Let $L=\mathfrak{o} x_{1}+\cdots+\mathfrak{b} x_{n}$. It follows from the uniqueness of the representation of elements with respect to a basis that $a_{1} x_{1}+\cdots+$ $a_{n} x_{n}$ runs through a complete set of representatives for $L / L(m)$ as $a_{1}, \cdots, a_{n}$ each runs through a complete set of representatives for $\mathrm{o} / \mathrm{o}(m)$.

Lemma 3.1. For $\chi \in X$ and $L \cong\left(\begin{array}{cc}\pi^{s} \\ a & 0\end{array}\right)$,

$$
\chi(L)=2^{f m} \sum_{\alpha \bmod 0(s)} \chi\left(\alpha^{2} \alpha \pi^{2 m-2 s}\right)
$$

if $s \leqq m$ and $\chi(L)=2^{f m} \sum_{\alpha \bmod (m)} \chi\left(\alpha^{2} a\right)$ if $m<s$.

Proof. Keeping in mind the preceding comment, $\chi(L)=$ $\sum_{\alpha, \beta} \chi\left(\alpha^{2} a+\alpha \beta \pi^{s}\right)$ with $\alpha \bmod \mathfrak{D}(m)$ and $\beta \bmod \mathfrak{D}(m)$. For a fixed $\alpha$, by [3, (1.6)], $\sum_{\beta} \chi\left(\alpha \beta \pi^{s}\right)=\left\{\begin{array}{l}2^{f m} \text { if ord } \alpha+s \geqq m \\ 0 \text { otherwise }\end{array}\right.$ with $\beta \bmod \mathfrak{o}(m)$. Therefore $\chi(L)=2^{f m} \sum \chi\left(\alpha^{2} a\right)$ with $\alpha \bmod \mathrm{o}(m)$ and ord $\alpha \geqq m-s$.

Since the case $s>m$ is immediate, assume $s \leqq m$. As $\gamma$ runs through a complete set of representatives for $\mathrm{o} / \mathrm{o}(s), \alpha=\gamma \pi^{m-s}$ runs through a complete set of those representatives for $\mathrm{o} / \mathrm{o}(m)$ such that ord $\alpha \geqq m-s$. Thus $\chi(L)=2^{f m} \sum_{\gamma} \chi\left(\gamma^{2} a \pi^{2 m-2 s}\right)$.

Proposition 3.2. For $\chi \in X$ and $H$ an i-hyperbolic plane, $\chi(H)=2^{f \min (2 m, m+i)}$.

Proof. This follows from Lemma 3.1 and the fact that $\chi(0)=1$.

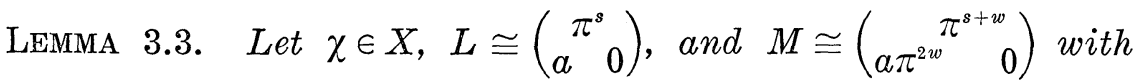
$0 \leqq w \leqq s+w \leqq m$. Then $\chi(M)=2^{f w} \chi(L)$.

Proof. Using Lemma 3.1, $\chi(M)=2^{f m} \sum \sum \chi\left(\left(\alpha+\beta \pi^{s}\right)^{2} a \pi^{2 m-2 s}\right)=$ $2^{f(m+w)} \sum \chi\left(\alpha^{2} \alpha \pi^{2 m-2 s}\right)=2^{f w} \chi(L)$ with $\alpha \bmod \mathfrak{p}(s)$ and $\beta \bmod \mathfrak{p}(w)$. 
Proposition 3.4. Let $L \cong\left(\begin{array}{c}\pi^{s} \\ e \pi^{w}\end{array}\right)$ and $m \geqq 0$. Then the following hold:

(1) If $m \leqq w$ or $2 s-w \leqq m$, then $\chi(L)=2^{f \min (2 m, m+s)}$.

(2) If $w<m<2 s-w$ with $w$ and $m$ of opposite parity, then $\chi(L)=0$.

(3) If $w<m<2 s-w$ with $w$ and $m$ of the same parity, then there exists $\chi_{1}$ and $\chi_{2}$ in $X$ with $m\left(\chi_{1}\right)=m\left(\chi_{2}\right)=m$ such that $\chi_{1}(L)=0$ and $\chi_{2}(L) \neq 0$. Furthermore, if $m(\chi)=m$ and $\chi(L) \neq 0$, then $\chi(L)=2^{f \min (2 m, m+s)}$.

Proof. (1) If $m \leqq w, \chi(L)=\chi\left(H_{s}\right)$; otherwise use Lemma 3.1.

(2) Claim. If $\chi$ has maximal support $\mathrm{o}(m), r+m \equiv 1 \bmod 2$, and $j \geqq(m-r+1) / 2>0$, then $\sum_{\alpha} \chi\left(\alpha^{2} e \pi^{r}\right)=0$ with $\alpha \bmod \mathfrak{o}(j)$.

Justification. With $\beta \bmod \mathfrak{p}((m-r-1) / 2), \quad \gamma \bmod \mathfrak{p}(1), \quad$ and $\delta \bmod \mathrm{o}(j-(m-r+1) / 2), \quad \sum \chi\left(\alpha^{2} e \pi^{r}\right)=\sum_{\beta} \sum_{r} \sum_{\delta} \chi\left(\beta+\pi^{(m-r-1) / 2} \gamma+\right.$ $\left.\left.\pi^{(m-r+1) / 2} \delta\right)^{2} e \pi^{r}\right)=\left[\sum_{\beta} \chi\left(\beta^{2} e \pi^{r}\right)\right]\left[\sum_{\gamma} \chi\left(\gamma^{2} e \pi^{m-1}\right)\right]\left[\sum_{\delta} \chi\left(\delta^{2} e \pi^{m+1}\right)\right]=0 \quad$ since $\sum_{\gamma} \chi\left(\gamma^{2} e \pi^{m-1}\right)=\sum_{\gamma} \chi_{(m-1)}(\gamma)=0$, and the claim holds.

If $s \leqq m$, take $r=2 m-2 s+w$ and $j=s$; and if $m<s$, take $r=w$ and $j=m$.

(3) If $s<m$, then by Lemma $3.3 L$ may be replaced by $\left(e \pi^{w+2 m-2 s} \pi^{m} 0\right)$. Hence we assume $m \leqq s$. Suppose $\chi(L)=0$ for all $\chi$ with $m(\chi)=m$. Let $M \cong\left(\begin{array}{c}\pi^{s} \\ e \pi^{w} \pi^{m-1}\end{array}\right)$. For $\chi \in X(m), \chi(M)=\chi(L)$. By [4, Proposition 3], $L\left(\pi^{m-1} ; \pi^{m}\right)=M\left(\pi^{m-1} ; \pi^{m}\right)$. Thus $\pi^{m-1} \in Q(L)$ which is impossible. Hence there exists $\chi_{2}$ with $m\left(\chi_{2}\right)=m$ and $\chi_{2}(L) \neq 0$.

Now suppose $\chi(L)$ is nonzero for all $\chi$ with $m(\chi)=m$. Write $N \cong\left(\begin{array}{c}\pi^{s} \\ e \pi^{m-1} 0\end{array}\right)$ and $N^{\prime} \cong\left(\begin{array}{ccc}e \pi^{w}+e \pi^{m-1} & \pi^{s}\end{array}\right)$. Using [6, LT $\quad$ b)], $N \oplus$ $L \cong N^{\prime} \oplus L$. Now $e \pi^{w}+e \pi^{m-1}$ is a unit times $\pi^{w}$ and therefore by the argument just gone through for $L, \chi\left(N^{\prime}\right) \neq 0$ for some $\chi$ with $m(\chi)=m$. Thus $\chi(N) \neq 0$; but this contradicts (2). Therefore there exists $\chi_{1}$ with $m\left(\chi_{1}\right)=m$ and $\chi_{1}(L)=0$.

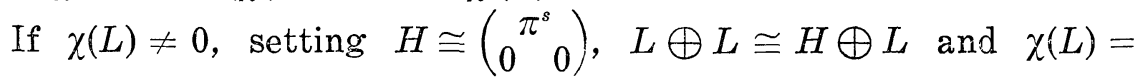
$\chi(H)=2^{f \min (2 m, m+s)}$.

It now follows that if $\chi(L) \neq 0$ then $\chi(L)= \pm 2^{n}$ for some nonnegative integer $n$.

\section{A reduction.}

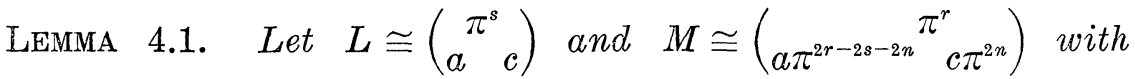
$r \geqq s+n$ and $n \geqq 0$. For any $\chi \in X$ such that $\chi\left(\begin{array}{cc}\pi^{s+n} \\ a & 0\end{array}\right), \chi\left(\begin{array}{cc}\pi^{s} \\ 0 & c\end{array}\right)$, 
and $\chi\left(\begin{array}{cc}\pi^{r} \\ 0 \quad c \pi^{2 n}\end{array}\right)$ are all nonzero, $\operatorname{sgn}(\chi(L))=\operatorname{sgn}(\chi(M))$, and $\chi(L)$ and $\chi(M)$ are both nonzero.

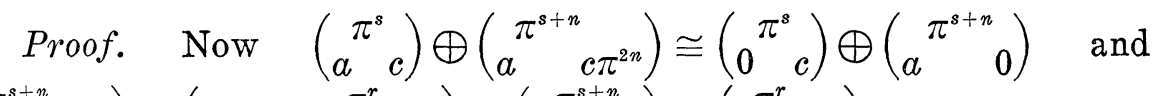

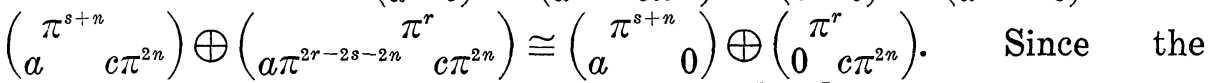
Gauss sum of the lattice on the right-hand side in each isometry is positive, the result follows.

THEOREM 4.2. Let $L=\sum_{i=0}^{t} L_{i}$ and $M=\sum_{i=0}^{t^{\prime}} M_{i}$ be canonical decompositions for non-defective spaces $L$ and $M$ respectively, and let $u=\min \left(u_{L}(s(t)), u_{M}\left(s\left(t^{\prime}\right)\right)\right)$. If $L$ and $M$ are of the same type $(I, T, R)$, and if $\chi(L)=\chi(M)$ for all $\chi \in X(2 T-u+1)$, then $\chi(L)=$ $\chi(M)$ for all $\chi \in X$.

Proof. Fix $\chi \in X$ such that $m>2 T-u+1$. Write $m=2 T-$ $u+2 n+j$ where $j=0$ or $j=1$. In view of Lemma $1.5 \operatorname{rank} L=$ rank $M$ and $s_{L}(i)=s_{M}(i)$ for $0 \leqq i \leqq t$. Fix $i$. Adjoining hyperbolic planes if necessary, decompose $L$ according to Lemma 1.2. Applying Proposition 3.2 to the hyperbolic part and Lemma 4.1 and Proposition 3.4 to the rest, we conclude that $\chi(L)=2^{f n \operatorname{rank} L} \chi_{(2 n)}(L)$. Similarly $\chi(M)=2^{f n \operatorname{rank} M} \chi_{(2 n)}(M)$.

\section{Classification of modular lattices.}

Proposition 5.1. If $s L=s M=\mathfrak{o}(s)$ and $\chi(L)=\chi(M)$ for all $\chi \in X(s)$, then $q(L)=q(M)$.

Proof. Case 1. $s=0$. Using the fact that $L$ has an 0 -modular orthogonal summand and Lemma 1.2, $q(L)=\mathfrak{v}^{2}+\pi \mathfrak{D}^{2}=q(M)$.

Case 2. $s \geqq 1$. First assume there exists $\chi \in X$ such that $m(\chi)=s$ and $\chi(L) \neq 0$. Then $\operatorname{sgn}(\chi(L)) 2^{\text {fm rank } L}=\chi(L)=\chi(M)=$ $\operatorname{sgn}(\chi(M)) 2^{f m \text { rank } M}$. Hence $\operatorname{rank} L=\operatorname{rank} M$, and by [4, Proposition 3] $L\left(\alpha ; \pi^{s}\right) M\left(\alpha ; \pi^{s}\right)$ for all $a \in \mathfrak{0}$. It follows that $q(L)=q(M)$.

Now assume $\chi(L)=\chi(M)=0$ for all $\chi \in X$ with $m(\chi)=s$. Assume without loss of generality that $\operatorname{rank} L \leqq \operatorname{rank} M$. Let $L^{\prime} \cong L \oplus H \oplus N$ such that $\operatorname{rank} L^{\prime}=\operatorname{rank} M, H$ is an $s$-hyperbolic lattice or 0 , and $N \cong\left(\pi^{s}\right)$ or 0 . Then $q\left(L^{\prime}\right)=q(L)$ and $\chi\left(L^{\prime}\right)=0$ for all $\chi \in X(s)$. Hence $\chi\left(L^{\prime}\right)=\chi(M)$ for all $\chi \in X(s)$. Then [4, Proposition 3] is applicable and it follows that $q(L)=q\left(L^{\prime}\right)=q(M)$.

Proposition 5.2. Let $\chi \in X$ with $m \geqq 1$, and let $L \cong$ 


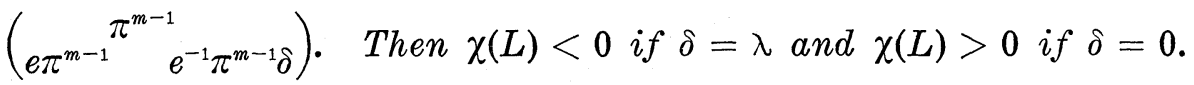

Proof. If $e \neq 1$ set $J \cong L \oplus H_{m-1}$. By Lemma 1.2 and the uniqueness of $\Delta(k J), J \cong\left(\begin{array}{cc}\pi^{m-1} & \pi^{m-1} \delta\end{array}\right) \oplus H_{m-1}$. Thus we may reduce to the case in which $e=1$. And since $\chi(L)=2^{2 f(m-1)} \chi_{(m-1)}\left(\begin{array}{c}1 \\ 1 \lambda\end{array}\right)$, we assume $m=1$.

If $\delta=0$ use Proposition 3.4. Assume $\delta=\lambda$. Fix a basis $\left\{p_{1}, \cdots, p_{f}\right\}$ for $\Omega$ over $\{0,1\}$. With $a_{1}, \cdots, a_{f}$ running through $\{0,1\}$, $\chi(L)=\sum_{a_{1}} \cdots \sum_{a_{f}}\left[\chi\left(\left(a_{1} p_{1}+\cdots+a_{f} p_{f}\right)^{2} \lambda\right)\right]$

$$
\left[1+\chi\left(p_{1}^{2}+a_{1} p_{1}^{2}+\cdots+a_{f} p_{1} p_{f}\right)\right] \cdots\left[1+\chi\left(p_{f}^{2}+a_{1} p_{1} p_{f}+\cdots+a_{f} p_{f}^{2}\right)\right] .
$$

Since $m=1$, at least one element in each basis for $\Omega$ must be mapped to -1 by $\chi$. Since $\lambda \notin \mathscr{P} \Omega$, it follows from elementary linear algebra that if $\left\{a_{1}, \cdots, a_{f}\right\} \subseteq\{0,1\}, \quad\left\{\left(a_{1} p_{1}+\cdots+a_{f} p_{f}\right)^{2}\right.$, $\left.p_{1}^{2}+a_{1} p_{1}^{2}+\cdots+a_{f} p_{1} p_{f}, \cdots, p_{f}^{2}+a_{1} p_{1} p_{f}+\cdots+a_{f} p_{f}^{2}\right\}$ contains a basis for $\Omega$. Thus $\chi(L)<0$.

Proposition 5.3. Let $L$ be an $s$-modular lattice with rank $L \geqq 4$ and $q(L)=e\left(\pi^{u} \mathfrak{D}^{2}+\pi^{v} \mathfrak{D}^{2}\right)$. Write $M \cong\left(\begin{array}{cc}\pi^{s} \\ e \pi^{u} & 0\end{array}\right)$ and $N \cong\left(\begin{array}{cc}\pi^{s} \\ e \pi^{v} & 0\end{array}\right)$. For $\chi \in X, \chi(L)=0$ iff $\chi(M)=0$ or $\chi(N)=0$.

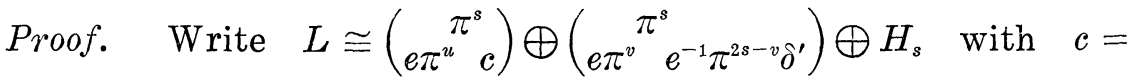
$e^{-1} \pi^{2 s-u} \delta+e^{-1} \pi^{v} a^{2}$ as in Lemma 1.2.

Sufficiency is obvious, so assume $\chi(L)=0$. Set $M^{\prime} \cong\left(\begin{array}{cc}\pi^{s} \\ c & 0\end{array}\right)$ and $N^{\prime} \cong\left(\begin{array}{rr}\pi^{s} \\ e^{-1} \pi^{2 s-v} \delta^{\prime} & 0\end{array}\right)$. By Propositions 3.2 and $3.4 \chi\left(N^{\prime}\right) \neq 0$. Thus at least one of $\chi(M), \chi(N)$, and $\chi\left(M^{\prime}\right)$ is zero. If $\chi\left(M^{\prime}\right)=0$, by Proposition 3.4 either $\chi(M)=0$ or $\chi(N)=0$.

THEOREM 5.4. Let $L_{1}$ and $L_{2}$ be s-modular lattices. $L_{1} \cong L_{2}$ iff $\chi\left(L_{1}\right)=\chi\left(L_{2}\right)$ for all $\chi \in X\left(2_{s}-\max \left(u_{L_{1}}, u_{L_{2}}\right)+1\right)$.

Proof. Necessity is obvious, so we assume $\chi\left(L_{1}\right)=\chi\left(L_{2}\right)$ for $\left.\chi \in X\left(2 s-\max u_{L_{1}}, u_{L_{2}}\right)+1\right)$. By Proposition 5.1, $u_{L_{1}}=u_{L_{2}}$. Call the common value $u$. By Propositions 3.4 and 5.3, $\chi\left(L_{i}\right)=$ $\operatorname{sgn}\left(\chi\left(L_{i}\right)\right) 2^{f(m+s)\left(\operatorname{rank} L_{i}\right) / 2}$ when $m=2 s-u+1$. Thus $\operatorname{rank} L_{1}=\operatorname{rank} L_{2}$. Adjoining the appropriate hyperbolic plane to $L_{1}$ and $L_{2}$ and applying Lemma 1.2 and Proposition 5.1, $Q\left(L_{1}\right)=q\left(L_{1}\right)=q\left(L_{2}\right)=$ $Q\left(L_{2}\right)=e\left(\pi^{u} \mathfrak{D}^{2}+\pi^{v} \mathfrak{D}^{2}\right)$ and

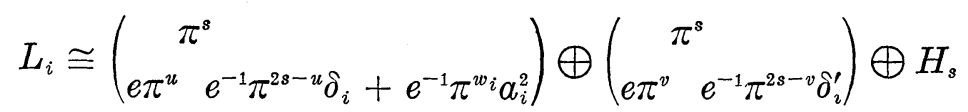


with $a_{i}$ a unit or zero, $w_{i} \geqq v$, and $w_{i}+v \equiv 0 \bmod 2$. By showing that ord $\left(\pi^{w_{1}} a_{1}^{2}+\pi^{w_{2}} a_{2}^{2}\right)>2 s-u$ and $\delta_{1}=\delta_{2}$, it will follow from [6,

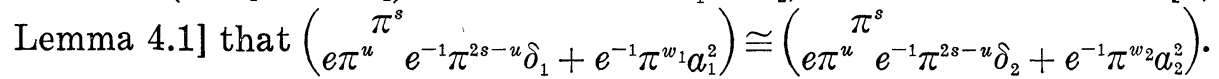
If we also show that $\delta_{1}^{\prime}=\delta_{2}^{\prime}$, we will have $L_{1} \cong L_{2}$.

By [6, Theorem 4.6],

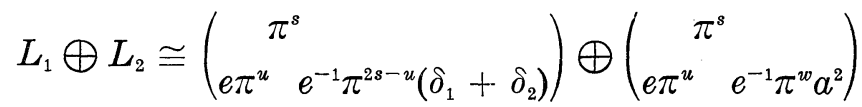

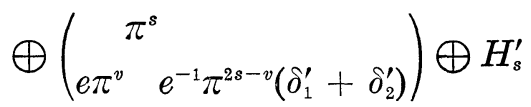

where $\pi^{w} a^{2}=\pi^{w_{1}} a_{1}^{2}+\pi^{w_{2}} a_{2}^{2}$ with $w \geqq v, w+v \equiv 0 \bmod 2$, and $a$ is

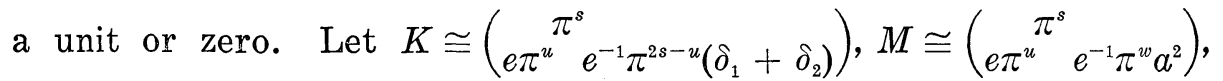
and $N \cong\left(\begin{array}{c}\pi^{s} \\ \pi^{v}\end{array} e^{-1} \pi^{2 s-v}\left(\delta_{1}^{\prime}+\delta_{2}^{\prime}\right)\right)$. For $\chi \in X \quad$ with $m=2 s-u$, $\chi\left(K \oplus M \oplus N \oplus H_{s}^{\prime}\right)=\chi\left(L_{1} \oplus L_{2}\right)>0 \quad$ and $\quad \chi\left(K \oplus H_{s}\right)>0$. Thus $\operatorname{sgn}(\chi(M))=\operatorname{sgn}(\chi(N))$.

Claim. If ord $\left(e^{-1} \pi^{w} a^{2}\right) \leqq 2 s-u$, then there exists $\chi^{\prime}, \chi^{\prime \prime} \in X$ with $m\left(\chi^{\prime}\right)=m\left(\chi^{\prime \prime}\right)=2 s-u$ such that $\chi^{\prime}(M)>0$ and $\chi^{\prime \prime}(M)<0$.

Justification. Since $w+u \equiv 1 \bmod 2$, ord $\left(e^{-1} \pi^{w} a^{2}\right) \neq 2 s-u$.

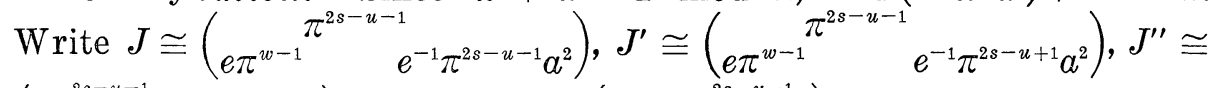
$\left(\begin{array}{cc}\pi^{2 s-u-1} & e^{-1} \pi^{2 s-u-1} a^{2}\end{array}\right)$, and $J^{\prime \prime \prime} \cong\left(\begin{array}{l}e \pi^{w-1} \pi^{2 s-u-1} \\ 0\end{array}\right) . \quad J \oplus J^{\prime} \cong J^{\prime \prime} \oplus J^{\prime \prime \prime}$. There exists $\chi^{\prime} \in X$ with $m=2 s-u$ such that $\chi^{\prime}\left(J^{\prime}\right)>0$ and $\chi^{\prime}\left(J^{\prime \prime} \oplus J^{\prime \prime \prime}\right)>0$. Hence $\chi^{\prime}(J)>0$. Taking $P \cong\left(\begin{array}{l}{ }_{e} \pi^{\pi^{2 s-u-1}} \\ 0\end{array}\right)$, by an argument like that given in the proof of Proposition 3.4 (3), $\chi^{\prime \prime}(J) \leqq 0$ for some $\chi^{\prime \prime} \in X$ with $m=2 s-u$. Now compare $M$ with $J$ by Lemma 4.1 to establish the claim.

It is easily seen that $\chi^{\prime}(N)=\chi^{\prime \prime}(N)$. Thus ord $\left(e^{-1} \pi^{w} a^{2}\right)>2 s-u$ and $\delta_{1}^{\prime}=\delta_{2}^{\prime}$.

By another application of Lemma 4.1, $\delta_{1}=\delta_{2}$.

6. Classification when $L=K(L)$. If $\operatorname{rank} L=1$, then $L \cong\left(e \pi^{u}\right)$. Otherwise $\operatorname{rank} L=2$, in which case $\operatorname{rank}_{\mathrm{o} 2} q(L)=2$ and $L \cong\left(e \pi^{u}\right) \oplus$ $\left(e \pi^{v}\right)$.

Theorem 6.1. Let $L=K(L), M=K(L)$, and $\operatorname{rank} L \leqq \operatorname{rank} M$. Write $w=\left\{\begin{array}{l}\max \left(u_{L}, u_{M}\right) \text { if } \operatorname{rank} L=\operatorname{rank} M=1 \\ \max \left(u_{L}, u_{M}, v_{M}\right) \text { if } \operatorname{rank} L=1 \text { and } \operatorname{rank} M=2 \\ \max \left(u_{L}, v_{L}, u_{M}, v_{M}\right) \text { if } \operatorname{rank} L=\operatorname{rank} M=2\end{array}\right.$ 
(1) If $\chi(L)=\chi(M)$ for $\chi \in X(w+1)$, then $\operatorname{rank} L=\operatorname{rank} M$.

(2) For $L \cong\left(e \pi^{u_{L}}\right)$ and $M \cong\left(e^{\prime} \pi^{u_{M}}\right), L \cong M$ if and only if $e \pi^{u_{L}} \in e^{\prime} \pi^{u_{M} k^{2}}$ and $\chi(L)=\chi(M)$ for all $\chi \in X(w)$.

(3) If $\operatorname{rank} L=\operatorname{rank} M=2$, then $L \cong M$ if and only if $\chi(L)=$ $\chi(M)$ for all $\chi \in X(w)$.

Proof. Write $M \cong\left(e^{\prime} \pi^{u_{M}}\right)$ or $M \cong\left(e^{\prime} \pi^{u_{M}}\right) \oplus\left(e^{\prime} \pi^{v_{M}}\right)$ and $L \cong\left(e \pi^{u_{L}}\right)$ or $L \cong\left(e \pi^{u}\right) \oplus\left(e \pi^{v} L\right)$ depending on the case at hand. Assume that $\chi(L)=\chi(M)$ for all $\chi \in X(w)$ and that $u_{L} \leqq u_{M}$. Setting $L^{\prime} \cong\left(e \pi^{u} L\right)$ and using (3.2) and Proposition 3.4, $u_{M}=u_{L}$.

(1) Assume rank $M=2$. Then $v_{M}$ exists, and comparing $L^{\prime}$ and $\left(e^{\prime} \pi^{v} M\right)$ with appropriate lattices of $\operatorname{rank} 2$, we see that $\chi\left(L^{\prime}\right) \neq 0$ for some $\chi$ with $m=v_{M}+1$ and $\chi\left(e^{\prime} \pi^{v_{M}}\right)=0$ for all $\chi$ with $m=$ $v_{M}+1$. Hence $L^{\prime}$ and $L$ are not isometric, and $\operatorname{rank} L=2$.

(2) Since $u_{L}=u_{M}$, this is obvious.

(3) Necessity is obvious. $L\left(\alpha ; \pi^{w}\right)=M\left(a ; \pi^{w}\right)$ for all $a \in \mathfrak{D}$. By a straightforward calculation $q(L)=q(M)$, and by [6, Lemma 1.2] $v_{L}=v_{M}$. It now follows that $L \cong M$.

\section{Classification when $L \neq K(L)$.}

Proposition 7.1. Let $L=\left(\sum_{i=0}^{t} L_{i}\right) \oplus K(L)$ be a saturated decomposition for the normal lattice $L$. For any $\chi \in X$ such that $\chi\left(L_{0}\right)=0, \chi\left(L_{0}^{\perp}\right) \geqq 0$.

Proof. Suppose $\chi\left(L_{0}\right)=0$ and $\chi\left(L_{0}^{\perp}\right) \neq 0$ for some $\chi \in X$. Using the hypothesis that the given decomposition is saturated and [6, Definition 5.1], there exists a unit $e$ such that $Q\left(L_{j}\right)=e\left(\pi^{u(s(j))}\right) 0^{2}+$ $\left(\pi^{v(s(j))} D^{2}\right)$ for $j=0,1$. By Propositions 5.3 and 3.4, $u(s(0))<m$ $2 s(0)-u(s(0))$.

We show that $m \leqq v(s(1))$. Then $m \leqq v(s(i))$ and hence $\chi\left(L_{i}\right) \geqq 0$ for $1 \leqq i \leqq t$. Since $\chi(K(L)) \geqq 0$, we then have $\chi\left(L_{0}^{\perp}\right)>0$.

Assume $v(s(1))<m$. Since $\chi\left(L_{1}\right) \neq 0$ and $u(s(1))<m<2 s(1)-$ $u(s(1)), \quad 2 s(0)-v(s(0)) \leqq 2 s(1)-v(s(1)) \leqq m$. So $\chi\left(e \pi^{u(s(0))} \pi^{s(0)}\right)=0$. By [6, Definition 5.1], one of $u(s(1))$ and $v(s(1))$ equals either $u(s(0))$

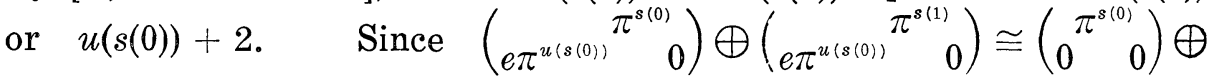
$\left(e \pi^{u(s(0))} \pi^{s^{(1)}} 0\right), \chi\left(e \pi^{u(s(0))} \pi^{\pi^{s(1)}} 0\right)=0$; and by Lemma 3.3, if $s(1) \leqq m$, $\chi\left(e \pi^{u(s(0))+2} \pi^{s(1)} 0\right)=0$. Thus $\chi\left(L_{1}\right) \neq 0$ implies $s(1)>m$. Hence $s(1)>$ $m \geqq 2 s(1)-v(s(1)) \geqq s(1)-1$. So $v(s(1)) \geqq m$.

Proposition 7.2. Let $L=\left(\sum_{i=0}^{t} L_{i}\right) \oplus K(L)=\left(\sum_{i=0}^{t} M_{i}\right) \oplus K(L)$ be canonical decompositions of a normal lattice $L$. If $u(j)=$ 
$u(j-1)+2$ and $v(j)=v(j-1)+2$ for some $j$ with $I<j \leqq T$, write $\sum_{i=0}^{j-I-1}\left(\Delta\left(k L_{i}\right)+\Delta\left(k M_{i}\right)\right)=\beta^{2} \pi^{-1}+\delta+\mathscr{P} k$ with $\beta \in \Omega\left[\pi^{-1}\right]$ and $\delta \in\{0, \lambda\}$. Then $\beta$ and $\delta$ are uniquely determined by the two decompositions and ord $\left(\beta^{2} \pi^{-1}+\delta\right) \geqq u(j)+v(j)-2 j-1$.

Proof. The uniqueness of $\beta$ and $\delta$ follows from [6, Lemma 1.1].

By [6, Theorems 2.2 and 2.3 and Lemma 1.2], a reduction can be made to the case in which the two canonical decompositions are complete and the second is obtained from the first by an elementary lattice transformation.

Denote $u(j-1), v(j-1)$, and $j-I$ by $u, v$, and $w$, respectively. Let $T_{r}$ denote the elementary lattice transformation used to obtain the second decomposition from the first. Only the cases for which $r=4$ and $r=9$ need be considered.

Case 1. $r=9$. If the two planes effected by $T_{9}$ are of the same modularity, the result holds trivially. If not, a reduction can be made to the case in which $T_{9}$ acts on $L_{w-1}$ and $L_{w}$. Then for some $c, d \in Q\left(L_{w-1}\right), \quad c^{\prime}, d^{\prime} \in Q\left(L_{w}\right)$, and $\alpha \in \mathfrak{D}, T_{9}$ sends $\left(\begin{array}{cc}\pi^{j-1} \\ c\end{array}\right) \oplus$ $\left(\begin{array}{c}\pi^{j} \\ c^{\prime}\end{array} d^{\prime}\right)$ to $\left(c+\alpha^{2} c^{\prime}{ }^{j-1} d\right) \oplus\left(\begin{array}{c}\pi^{j} \\ c^{\prime}\end{array} d^{\prime}+\alpha^{2} \pi^{2} d\right)$ and leaves the other planes fixed.

By a direct computation, $\sum_{i=0}^{w-1}\left(\Delta\left(k L_{i}\right)+\Delta\left(k M_{i}\right)\right)=\alpha^{2} c^{\prime} d \pi^{-2 j+2}+$ $\mathscr{P} k=\beta^{2} \pi^{-1}+\delta+\mathscr{P} k$ with ord $\left(\beta^{2} \pi^{-1}+\delta\right) \geqq u(j)+v(j)-2 j-1$, where $\beta \in \Omega\left[\pi^{-1}\right]$ and $\delta \in\{0, \lambda\}$.

Case 2. $r=4$. If the plane altered by the application of $T_{4}$ has modularity greater that $j-1$, the result follows. Otherwise there exists a sequence of complete decompositions from the first given decomposition to the second, each of which can be obtained from its predecessor by either an application of $T_{9}$ or an application of $T_{4}$ to a plane of modularity $j-1$. Assume $T_{4}$ is applied to a plane of modularity $j-1$. For some $c, d \in Q\left(L_{w-1}\right), c^{\prime} \in Q(K(L))$, and $\alpha \in \mathfrak{D}, T_{4}$ sends $\left(\begin{array}{cc}\pi^{j-1} \\ c & d\end{array}\right)$ to $\left(\begin{array}{c}\alpha^{2} c^{\prime} \\ \pi^{j-1}\end{array}\right)$ and leaves all else fixed. Now $c^{\prime} \in Q\left(L_{w}\right)$; so the proof follows exactly as in Case 1 .

Notation. Let $L$ and $M$ be lattices of the same type $(I, T, R)$, $j$ be a rational integer such that $I<j<T$, and $H=\sum_{i=1}^{T} H_{i}$ with $H_{i} i$-hyperbolic of rank 8. $L \oplus H$ and $M \oplus H$ are normal lattice of type $(I, T, R+R(H))$; and if $\left(\sum_{i=0}^{t} L_{i}\right) \oplus K(L \oplus H)$ and $\left(\sum_{i=0}^{t^{\prime}} M_{i}\right) \oplus$ $K(M \oplus H)$ are canonical decompositions of $L \oplus H$ and $M \oplus H$ respectively, then $t=t^{\prime}=T-I$ and $s\left(L_{i}\right)=s\left(M_{i}\right)=I+i$ for $0 \leqq i \leqq t$. Write 


$$
\begin{aligned}
\operatorname{Ord}(L, M, j) & =\min \left\{\operatorname{ord}\left(\beta^{2} \pi^{-1}+\delta\right) \mid \beta^{2} \pi^{-1}+\delta+\mathscr{P} k\right. \\
& =\sum_{i=0}^{j-I-1}\left(\Delta\left(k L_{i}\right)+\Delta\left(k M_{i}\right)\right)
\end{aligned}
$$

for some canonical decompositions $\left(\sum_{i=0}^{T-I} L_{i}\right) \oplus K(L \oplus H)$ and $\left(\sum_{i=0}^{T-I} M_{i}\right) \oplus K(M \oplus H)$ of $L \oplus H$ and $M \oplus H$ respectively

LEMMA 7.3. Let $L \cong\left(\begin{array}{c}\pi^{s} \\ a\end{array}\right)$ and $M$ be an $s+1$-modular lattice such that $\operatorname{rank} M \geqq 8$ and $\pi^{2} q(L) \subseteq q(M)$. It $a+a^{\prime} \in q(M)$, then $L \oplus M \cong\left(\begin{array}{c}\pi^{s} \\ a^{\prime}\end{array}\right) \oplus M^{\prime}$, where $M^{\prime}$ is $s+1$-modular and $q\left(M^{\prime}\right)=q(M)$.

The proof is a straightforward manipulation of lattices.

Lemma 7.4. Let $s, w$, and $w^{\prime}$ be nonnegative integers such that either $w^{\prime}=w$ or $w^{\prime}=w+2$; and let $e$ be a unit. For any $\chi \in X$ with $m>s$ and $\chi\left(e \pi^{w^{s}} 0\right)=0, \chi\left(e \pi^{w^{\prime}}{ }^{\pi^{s+1}} 0\right)=0$.

Proof. If $w^{\prime}=w+2$, use Lemma 3.3. If $w^{\prime}=w$, write

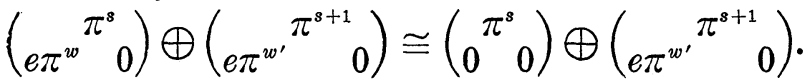

THEOREM 7.5. For lattices $L$ and $M, L \cong M$ if and only if

(1) $L$ and $M$ are of the same type $(I, T, R)$;

(2) For $I \leqq i \leqq T, q\left(L(i) \oplus H_{i}\right)=q\left(M(i) \oplus H_{i}\right)$. That is, the $i$ th norm groups of $L$ and $M$ are the same.

(3) $\chi(L)=\chi(M)$ for all $\chi \in X(2 T-u(T)+2)$;

(4) If for some $j$ with $I<j \leqq T, u(j)=u(j-1)+2$ and $v(j)=v(j-1)+2$, then $\operatorname{Ord}(L, M, j) \geqq u(j)+v(j)-2 j-1$;

(5) If $K(L) \cong\left(d_{1}\right)$ and $K(M) \cong\left(d_{2}\right)$, then $d_{1} \in d_{2} k^{2}$.

Proof. Necessity. Condition (4) follows from [6, Lemma 5.2] and Proposition 7.2. The others hold trivially.

Sufficiency. Replacing $L$ and $M$ by $L \oplus\left(\sum_{i=I}^{T} H_{i}\right)$ and $M \oplus$ $\left(\sum_{i=I}^{T} H_{i}\right)$ respectively, we may assume $L$ and $M$ are normal lattices. Proceed by induction on $n=$ length $d=$ length $M$.

Part I. $n=1$. Then $T=I$. Write $u(I)=u$ and $v(I)=v$.

Case 1. $\operatorname{Rank}(K(L))=0$. Assume $K(M) \neq 0$. Then $u=u_{K(M)}$ by normality; and for any unit $e$ and any $\chi$ with $m=2 T-u+1$, $\chi\left(e \pi^{u} K(M)=0\right.$. Thus $\chi(L)=\chi(M)=0$ for such $\chi$. But $\chi(L)=0$ by Propositions 3.4 and 5.3. Therefore $K(M)=0$. It now follows from 
Theorem 5.4 that $L \cong M$.

Case 2. $\operatorname{Rank}(K(L))=1$. By normality, $K(L) \cong\left(e \pi^{u}\right)$ for some unit $e$. Write $L \cong L_{0} \oplus K(L)$ with $q\left(L_{0}\right)=e\left(\pi^{u} \mathrm{D}^{2}+\pi^{v} \mathrm{D}^{2}\right)$. There exists $\chi \in X$ with $m=2 I-v+3$ and $\chi(L) \neq 0$. Since $\chi\left(e \pi^{v}\right)=0$, $\operatorname{rank}(K(M)) \neq 2$. Using condition (5), $K(M) \cong\left(e \pi^{u}\right)$. Using [6, Lemma

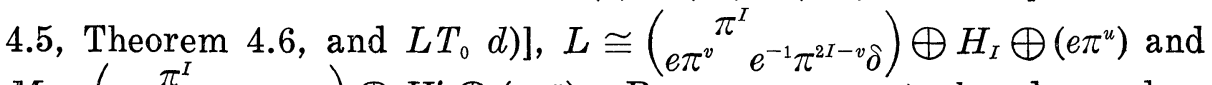
$M \cong\left(\begin{array}{c}\pi^{I} \\ e \pi^{v} e^{-I} \pi^{2 s-v} \delta^{\prime}\end{array}\right) \oplus H_{I}^{\prime} \oplus\left(e \pi^{u}\right)$. By an argument already used on several occasions (see e.g., Theorem 5.4) $L \cong M$.

Case 3. $\operatorname{Rank}(K(L))=2$. $K(L) \cong\left(e \pi^{u}\right) \oplus\left(e \pi^{v}\right)$ for some unit $e$. By an argument similar to the one given in Case $2, L \cong H_{I} \oplus$ $\left(e \pi^{v}\right) \oplus\left(e \pi^{v}\right) \cong M$.

Part II. Assume $n>1$ and that the result holds for normal lattices of length $n-1$. By [6, Lemma 5.3], $L$ and $M$ admit saturated decompositions $\left(\sum_{i=0}^{n-1} L_{i}\right) \oplus K(L)$ and $\left(\sum_{i=0}^{n-1} M_{i}\right) \oplus K(M)$ respectively. Our strategy is to show the following:

(7.1) For $j=0,1$, there exists $s(j)$-modular lattices $L_{j}^{*}$ and $M_{j}^{*}$ with $q\left(L_{j}^{*}\right)=q\left(L_{j}\right)$ and $q\left(M_{j}^{*}\right)=q\left(M_{j}\right)$ such that $L_{0} \oplus L_{1} \cong L_{0}^{*} \oplus L_{1}^{*}$, $M_{0} \oplus M_{1} \cong M_{0}^{*} \oplus M_{1}^{*}$, and $L_{0}^{*} \cong M_{0}^{*}$.

Then $\left(L_{0}^{*}\right)^{\perp}$ and $\left(M_{0}^{*}\right)^{\perp}$ are normal lattices of length $n-1$ which satisfy conditions (1), (2), (4), and (5). For any $\chi \in X(2 T-u(T)+2)$ such that $\chi\left(L_{0}^{*}\right) \neq 0, \quad \chi\left(\left(L^{*}\right)^{\perp}\right)=\chi\left(\left(M_{0}^{*}\right)^{\perp}\right)$. If $\chi \in X(2 T-u(T)+2)$ and $\chi\left(L_{0}^{*}\right)=0$, then using Lemma 7.4 and Proposition 5.3, $\chi\left(\left(L_{0}^{*}\right)^{\perp}\right)=$ $0=\chi\left(\left(M_{0}^{*}\right)^{\prime}\right)$. Thus condition (3) is satisfied. The result follows easily by induction.

We verify (7.1) by cases. But first write $L_{0} \cong L_{0}^{\prime} \oplus L_{0}^{\prime \prime} \oplus H_{I}$ where $L_{0}^{\prime} \cong\left(\begin{array}{ll}e \pi^{u(I)} \pi^{I} & e^{-1} \pi^{2 I-u(I)} \delta+e^{-1} \pi^{v(I)} a^{2}\end{array}\right) \oplus\left(e \pi^{v(I)} \pi^{I} e^{-1} \pi^{2 I-v(I)} \delta^{\prime}\right)$ and $L_{0}^{\prime \prime} \cong\left(\begin{array}{cc}e \pi^{u(I)} \pi^{I} & 0\end{array}\right) \oplus\left(\begin{array}{cc}e \pi^{v(I)} & \pi^{I} \\ 0\end{array}\right)$ with $\delta, \delta^{\prime} \in\{0, \lambda\}, a \in \mathfrak{p}$, and $e$ a unit such that $q_{s(j)}(L)=e\left(\pi^{u(s(j))} D^{2}+\pi^{v(s(j))} D^{2}\right)$ for $j=0,1$.

Case A. $v(I+1)=v(I)$. By [6, Definition 5.1], $u(I+1)=u(I)$ or $u(I)+2$. Applying Lemma 7.3 twice, $L_{0} \oplus L_{1} \cong L_{0}^{*} \oplus L_{i}^{*}$ with $L_{0}^{*} \cong L_{0}^{\prime \prime} \oplus H_{I}^{\prime} . \quad M_{0}^{*}$ and $M_{1}^{*}$ exist with $M_{0}^{*} \cong L_{0}^{\prime \prime} \oplus H_{I}^{\prime}$, and these lattices satisfy (7.1).

Case B. $v(I+1)=v(I)+1$. Then $u(I+1)=v(I)=u(I)+1$. If $u(I)<I$, the result follows as in Case A. Suppose $u(I)=I$. $L_{0}^{*}=L_{0} \cong\left(\begin{array}{cc}\pi^{I} \\ \pi^{I} & \pi^{I} \delta\end{array}\right) \oplus H_{I}^{\prime}$ and $M_{0}^{*} \cong\left(\begin{array}{cc}\pi^{I} \\ \pi^{I} & \pi^{I} \delta_{1}\end{array}\right) \oplus H_{I}^{\prime}$ and the result follows. 
Case C. $v(I+1)=v(I)+2$. If $v(I+1) \leqq I+1$ and $u(I+1)=$ $u(I), L_{0}^{*} \cong L_{0}^{\prime \prime} \oplus H_{I}^{\prime}$.

If $v(I+1) \leqq I+1$ and $u(I+1)=u(I)+2$, write $a=a_{1}+a_{2}$ with $a_{1} \in \Omega$ and $a_{2} \in \mathfrak{D}(1)$. In this case $L_{0}^{*} \cong\left(\begin{array}{c}e \pi^{u(I)} \pi^{I} \\ e^{-1} \pi^{v(I)} a_{1}^{2}\end{array}\right) \oplus L_{0}^{\prime \prime} \oplus H_{I}^{\prime}$

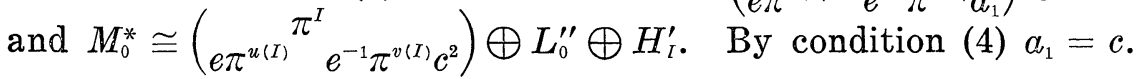

We are left with the situation in which $v(I+1)=I+2$. If $u(I+1)=u(I)$, take $L_{0}^{*} \cong\left(\begin{array}{c}e \pi^{v(I)} \pi^{I} \\ e^{-1} \pi^{2 I-v(I)} \delta^{\prime}\end{array}\right) \oplus L_{0}^{\prime \prime} \oplus H_{I}^{\prime}$ and $M_{0}^{*} \cong$ $\left({ }_{e \pi^{v(I)}} \pi^{I} e^{-1} \pi^{2 s-v(I)} \delta_{1}\right) \oplus L_{0}^{\prime \prime} \oplus H_{I}^{\prime}$. If $u(I+1)=u(I)+2$, by the argument used in the case where $v(I+1)=v(I)+2 \leqq I+1$ and $u(I+1)=u(I)+2$,

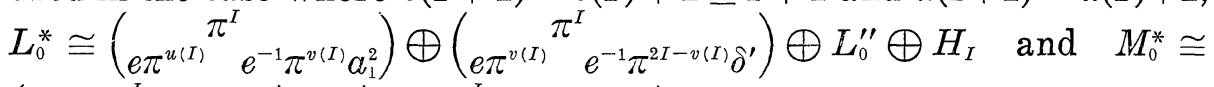

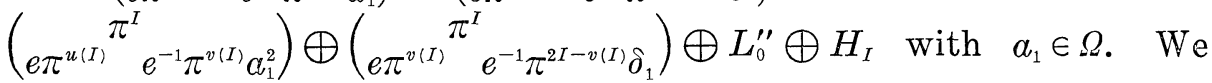
need only show that $\delta^{\prime}=\delta_{1}$. The difficulty here is in checking that there exists $\chi \in X$ with $m=I+1$ and $\chi(L) \neq 0$. Let $r$ be the largest integer such that $0 \leqq r \leqq n-1$ and $u(I+r)<I+1$. Since $u(I)<I+1, r$ exists. $u(I+r)+(I+1) \equiv 0 \bmod 2$. So there exists $\chi$ with $m=I+1$ and $\chi\left(e_{e_{s(r)} \pi^{u(s(r))}} \pi^{s(r)} 0\right) \neq 0$. It follows that $\chi\left(L_{r}\right) \neq 0$ and by induction on $r, \chi\left(\sum_{r=0}^{r} L_{i}\right) \neq 0$. Thus $\chi(L) \neq 0$.

\section{REFERENCES}

1. C. Arf, Untersuchungen über quadratische formen in körpern der charakteristik 2, I. J. Reine Angew. Math., 183 (1940), 148-167.

2. - Uber arithmetische aequivalenz quadratisher formen in palenzreihenkörpern über einem vollkommen körper der charakterisk 2, Rev. Fac. Sci. Univ. Istanbul, 8 (1943), 297-327.

3. R. Jacobowitz, Gauss sums and the local classification of hermitian forms, Amer.

J. Math., 90 (1968), 528-552.

4. O. T. O'Meara, Local characterization of integral quadratic forms by Gauss sums. Amer. J. Math., 79 (1957), 687-709.

5. —_ Introduction to Quadratic Forms, Springer-Verlag, Berlin (1963).

6. C.-H. Sah, Quadratic forms over fields of characteristic 2, Amer. J. Math., 82 (1960), 812-830.

Received December 30, 1975 and in revised form February 18, 1976. The results of this paper are contained in the author's doctoral thesis. The author wishes to express her appreciation to her advisor, Professor Ronald Jacobowitz.

Berea COLlege 



\section{PACIFIC JOURNAL OF MATHEMATICS}

\section{EDITORS}

RICHARD ARENS (Managing Editor) University of California

Los Angeles, California 90024

R. A. Beaumont

University of Washington

Seattle, Washington 98105
J. DUGUNDJI

Department of Mathematics University of Southern Californı

Los Angeles, California 90007

D. Gilbarg and J. Milgram

Stanford University

Stanford, California 94305

\section{ASSOCIATE EDITORS}
E. F. BECKENBACH
B. H. NeumanN
F. WOLF
K. YosHIDA

\section{SUPPORTING INSTITUTIONS}

UNIVERSITY OF BRITISH COLUMBIA

CALIFORNIA INSTITUTE OF TECHNOLOGY

UNIVERSITY OF CALIFORNIA

MONTANA STATE UNIVERSITY

UNIVERSITY OF NEVADA

NEW MEXICO STATE UNIVERSITY

OREGON STATE UNIVERSITY

UNIVERSITY OF OREGON

OSAKA UNIVERSITY
UNIVERSITY OF SOUTHERN CALIFORNIA

STANFORD UNIVERSITY

UNIVERSITY OF TOKYO

UNIVERSITY OF UTAH

WASHINGTON STATE UNIVERSITY

UNIVERSITY OF WASHINGTON

* * * *

AMERICAN MATHEMATICAL SOCIETY

NAVAL WEAPONS CENTER 


\section{Pacific Journal of Mathematics \\ Vol. 65, No. $1 \quad$ September, 1976}

David Lee Armacost, Compactly cogenerated LCA groups ............. 1

Sun Man Chang, On continuous image averaging of probability measures ...... 13

J. Chidambaraswamy, Generalized Dedekind $\psi$-functions with respect to a

polynomial. II................................... 19

Freddy Delbaen, The Dunford-Pettis property for certain uniform algebras ..... 29

Robert Benjamin Feinberg, Faithful distributive modules over incidence

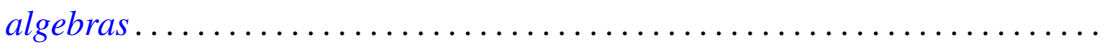

Paul Froeschl, Chained rings . . . . . . . . . . . . . . . . . . . .

John Brady Garnett and Anthony G. O'Farrell, Sobolev approximation by a sum

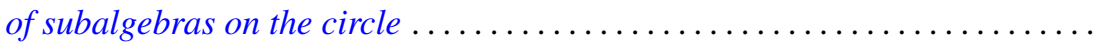

Hugh M. Hilden, José M. Montesinos and Thomas Lusk Thickstun, Closed

oriented 3-manifolds as 3-fold branched coverings of $S^{3}$ of special type.....

Atsushi Inoue, On a class of unbounded operator algebras ................

Peter Kleinschmidt, On facets with non-arbitrary shapes.

Narendrakumar Ramanlal Ladhawala, Absolute summability of Walsh-Fourier

series

Howard Wilson Lambert, Links which are unknottable by maps . . . . . . . . . . .

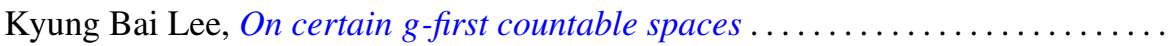

Richard Ira Loebl, A Hahn decomposition for linear maps .................

Moshe Marcus and Victor Julius Mizel, A characterization of functionals on $W_{1}^{p}$ possessing autonomous kernels. I . .

James Miller, Subordinating factor sequences and convex functions of several

variables.

Keith Pierce, Amalgamated sums of abelian l-groups ...

Jonathan Rosenberg, The $C^{*}$-algebras of some real and $p$-adic solvable

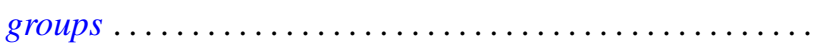

Hugo Rossi and Michele Vergne, Group representations on Hilbert spaces defined

in terms of $\partial_{b}$-cohomology on the Silov boundary of a Siegel domain . .

Mary Elizabeth Schaps, Nonsingular deformations of a determinantal

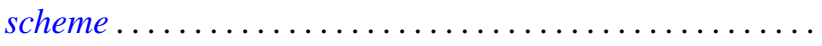

S. R. Singh, Some convergence properties of the Bubnov-Galerkin method...

Peggy Strait, Level crossing probabilities for a multi-parameter Brownian

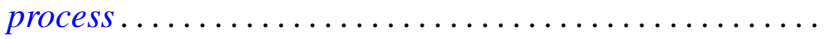

Robert M. Tardiff, Topologies for probabilistic metric spaces .

Benjamin Baxter Wells, Jr., Rearrangements of functions on the ring of integers of

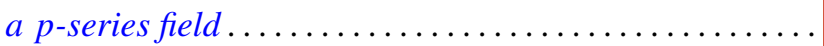

Robert Francis Wheeler, Well-behaved and totally bounded approximate identities for $C_{0}(X)$.

Delores Arletta Williams, Gauss sums and integral quadratic forms over local

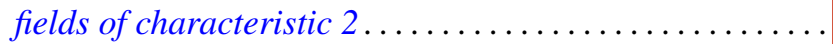

John Yuan, On the construction of one-parameter semigroups in topological 